\title{
Mesenteric extraskeletal Ewing sarcoma with small bowel involvement
}

\author{
Istvan Zöllei ${ }^{*}$, Zsolt Csobó ${ }^{1}, J^{\prime}$ nos Cifra ${ }^{2}$, Tamás Tornóczky ${ }^{3}$, Bálint Kaszás ${ }^{3}$ and Attila Győri ${ }^{1}$ \\ ${ }^{1}$ Department of Surgery, County Teaching Hospital, Szekszárd, Hungary \\ ${ }^{2}$ Department of Pathology, County Teaching Hospital, Szekszárd, Hungary \\ ${ }^{3}$ Institute of Patholology, University of Pécs, Hungary
}

\section{Introduction}

Ewing sarcoma (ES) is a malignant, small blue cell neoplasm of the bone and soft tissue, characterized by specific translocations involving the EWSR1 gene. It was originally described by James Ewing in 1921 . He observed that this highly aggressive, originally bone tumor was remarkably sensitive to radiation therapy. However extraskeletal cases are rare, several cases have been reported with different localisations, including - jejunum [1], chest wall [2], paravertebral region [3], stomach [4], lung parenchyma [5], breast tissue [6] and mesentery-ileocecum [7]. Soft tissue sarcomas encompass around $1 \%$ of all malignant tumors, but they are relatively more frequent in childhood and adolescent age [8], and extraskeletal Ewing sarcoma belongs to this group of tumors.

"The goal of this study" is to present a case report of an urgent surgery when explorative laparotomy was performed due to an acute abdominal emergency. During the surgery, a small bowel segment and an associated mesenteric mass were removed. Histological examination confirmed extraskeletal mesenteric Ewing sarcoma with small bowel involvement.

\section{Case history}

A 45 years old man arrived in the Department of Emergency of Janos Balassa County Teaching Hospital, Szekszard (Hungary) with fever and severe abdominal pain. The abdominal sonography described a bowel conglomerate and a non-specific mass next to the cecum. The routine laboratory tests did not confirm any severe inflammation. Explorative laparotomy was performed. During the surgery, a mesenteric mass of at least $4 \mathrm{~cm}$, and a small bowel segment of $15 \mathrm{~cm}$ were removed. Anastomosis of small bowel was performed, naturally. The patient was emitted on the 7 th postoperative day without any complication. The histological examination confirmed a rare extraskeletal Ewing sarcoma in the mesenterium that involved the small bowel wall. After the surgery adjuvant oncological treatment was administered.

\section{Histological and molecular pathology results}

The tissue samples revealed a small round cell tumor, originating from the mesenterium with the microscopic involvement of the small intestine (Figures 1 and 2). Immunohistochemical examination showed an extensive, diffuse CD117 and vimentin reaction with multifocal S100 positivity, while CD45, CD3, CD20, chromogranin-A, DOG1, KL1, synaptophysin, and CD56 were all negative. The CD99 was weak to moderate positive on the cytoplasmic membrane of the tumor cells

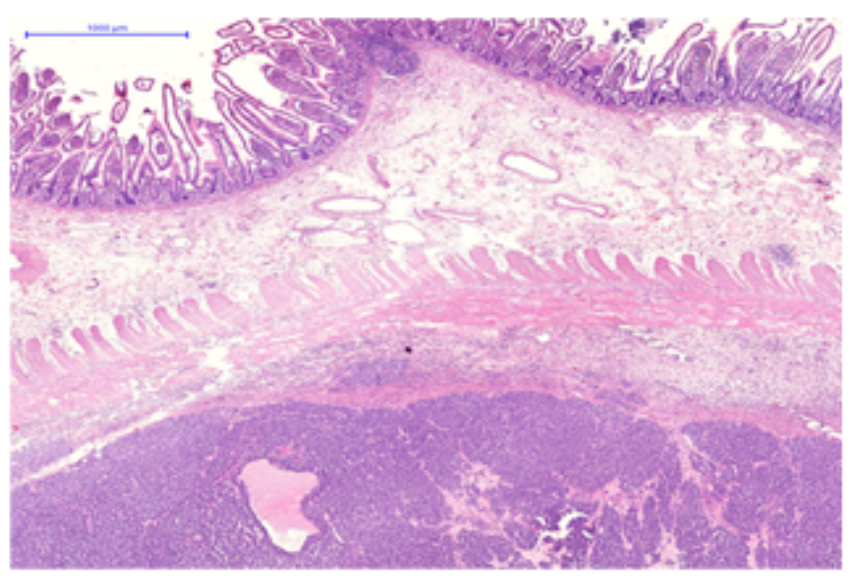

Figure 1. HE staining, low magnification: Sheets of tumor cells invading the muscular layer of the small intestine

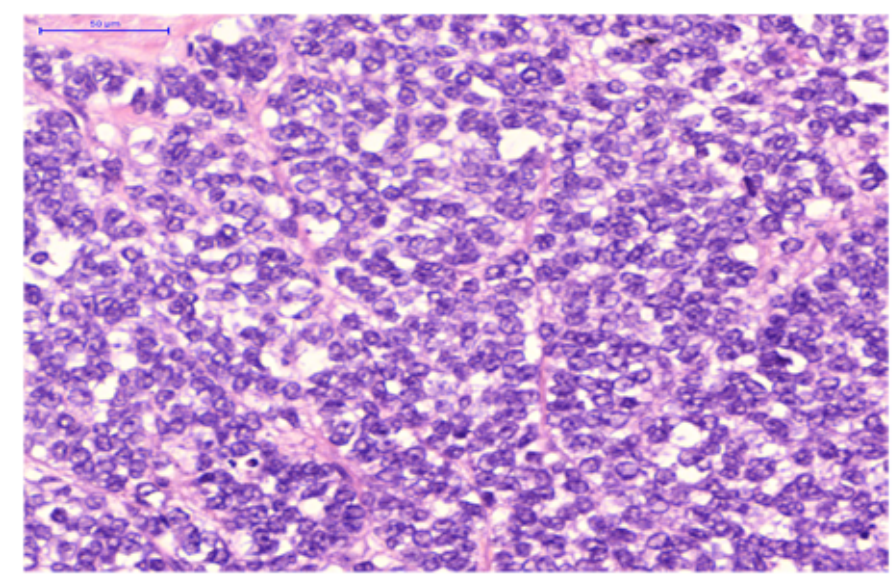

Figure 2. HE staining, high magnification: Characteristic hypercellular, small, round cell tumor. The cells arranged in large sheets and have hyperchromatic nuclei with inconspicuous nucleoli

${ }^{\star}$ Correspondence to: Istvan Zöllei, Department of Surgery, County Teaching Hospital, Szekszárd, Hungary, E-mail: dr.zollei.istvan@gmail.com

Received: June 27, 2020; Accepted: July 07, 2020; Published: July 10, 2020 


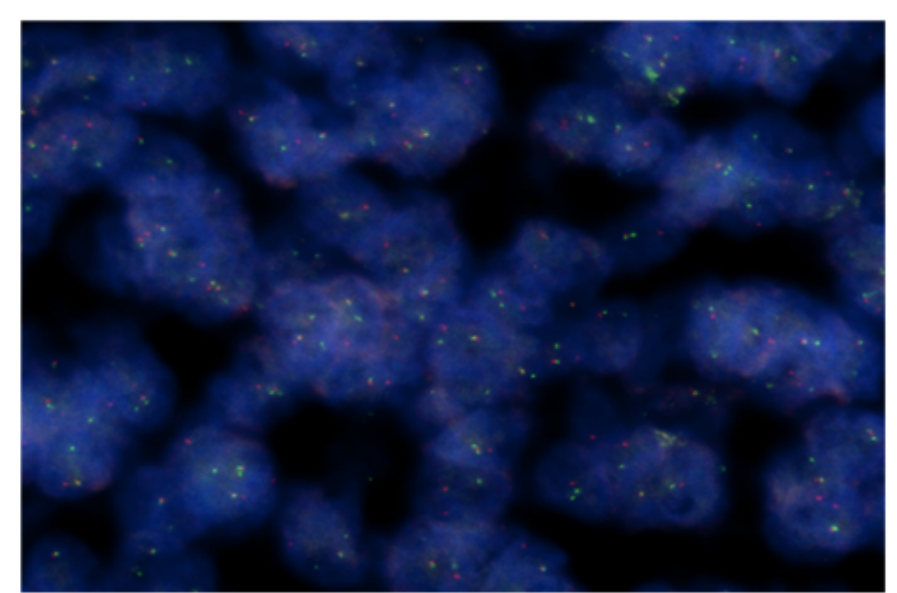

Figure 3. FISH demonstrates the split-up of the EWSR1 gene. Note the separated green and red signals representing the 3 ' and 5 ' sequences on the two sides of the breakpoint

beneath the exulcerated area. Mutational analysis of KIT (exons 9, 11, 13 , and 17) and PDGFRA (exon 18) genes revealed wild type in all exons tested. In addition, the EWSR1 fluorescent in situ hybridization (FISH) reaction confirmed the separation of the green and red signals (signal split-up), indicating the involvement of the EWSR1 gene by a translocation, confirming the diagnosis of the extraskeletal Ewing sarcoma (Figure 3).

\section{Discussion}

According to the latest WHO classification, published electronically in February 2020, Ewing sarcoma of the soft tissues and bone belongs to the group of „undifferentiated round cell sarcomas of bone and soft tissue" in conjunction with "round cell sarcoma of EWSR1-non-ETS fusion”, „CIC-rearranged-”, and „BCOR-rearranged sarcomas” [9]. Diagnosis of extraskeletal Ewing sarcoma in abdominal localization can be extremely challenging since this tumor is rare and the potential differential diagnostic alternatives are extensive. One must consider several mesenchymal neoplasms that involve the mesenteric tissues and the small bowel wall [10]. Using an immunohistochemical panel which includes myoid, lymphoid, and keratin markers one can potentially narrow the possible diagnoses. One of the most common nonepithelial neoplasms, arising in this localization is the gastrointestinal stromal tumor (GIST), which typically shows a c-kit (CD-117) positive immunohistochemical reaction. Ewing-sarcoma sometimes also shows significant cytoplasmic staining with this marker [11]. Evaluation of the CD99 expression also can cause a significant problem, when moderate, focal reaction is seen due to formalin fixation issues in large specimens. Moreover, CD99 can show diffuse, strong positivity in lymphoblastic lymphoma [12], which also may arise in abdominal localization. Clear cell sarcoma-like tumor of the mesentery (CCSLTM) is another a diagnostic alternative and only the "clear" phenotype of the tumor cells, the CD-117 negativity, and the consistently diffuse positivity for $\mathrm{S} 100$ protein distinguish them from extraskeletal ES. To confirm the diagnosis, EWSR1 FISH could be performed, especially if histology indicates a small, blue, round cell neoplasm with doubtful immunophenotype bearing in mind, that CCSLTM also shows EWSR1 split-up signal.

The best chance of the successful treatment of the disease is in special cancer centers and with early diagnosis. The complex therapy of Ewing sarcoma in the extraskeletal soft tissue and in the bone tumors is similar, but not the same. If the Ewing sarcoma has proved before the surgery, neoadjuvant chemotherapy could be the first choice of treatment. There are known three-drug therapy (cyclophosphamide, actinomycin-D, vincristine) and five drug therapy (vincristine, doxorubicin, cyclophophosphamide, ifosfamide, vincristine), also. The next step is the radical surgery (if it is possible). The decision of surgical extent is easier in the soft tissue surgery than it is in the cases of bone tumors. The radiotherapy used as neoadjuvant or/and postoperative adjuvant treatment. The radiotherapy is very useful in the treatment of Ewing sarcoma of bones, but it is not a suitable treatment for extraskeletal Ewing sarcoma of the small intestine (in the presented case). If the Ewing sarcoma diagnosis is proved after an urgent surgery the neoadjuvant therapy is not possible. In that cases oncoteam has to decide about the adjuvant chemo-radiotherapy.

\section{Conclusion}

Extraskeletal Ewing sarcoma is an extremely rare tumor. It is even more rare, when it locates in the mesentery, and appears in the form of an abdominal mass causing severe pain leading to urgent surgery. The exact diagnosis was established after the histological and molecular pathological examination of the surgical specimen. The resection of the mesenteric mass was graded R0 by the pathologist, which may be resulted in a symptom-free and long survival. The adjuvant chemotherapy may improve the postoperative outcome, but regular clinical and imaging control is recommended.

\section{References}

1. Cantu C, Bressier E, Dermawan J, Paral K (2019) Extrasceletal Ewing sarcoma of the jejunum: A case report. Perm J 23: 18-255. [Crossref]

2. Xie CF, Liu MZ, Xi M (2010) Extraskeletal Ewing's sarcoma: a report of 18 cases and literature review. Chin J Cancer 29: 420-424. [Crossref]

3. Ulusan S, Koc Z, Canpolat ET, Colakoglu T (2007) Radiological findings of primary retroperitoneal Ewing sarcoma. Acta Radiol 48: 814-818. [Crossref]

4. Maxwell AW, Wood S, Dupuy DE (2016) Primary extraskeletal Ewing sarcoma of the stomach: a rare disease is an uncommon location. Clin Imaging 40: 843-845. [Crossref]

5. Zhang J, Dong A, Cui Y, Wang Y (2019) FDG PET/CT in a case of primary pulmonary Ewing sarcoma. Clin Nucl Med 44: 666-668. [Crossref]

6. Srivastava S, Arora J, Parakh A, Goel RK (2016) Primary extraskeletal Ewing'sarcoma/ primitive neuroectoderma tumor of breast. Indian J Radiol Imaging 26: 226-230. [Crossref]

7. Peng L, Yang L, Wu N, Wu B (2015) Primary primitive neuroectodermal tumor arising in the mesentery and ileocecum: A report of three cases and review of the literature. Exp Ther Med 9: 1299-1303. [Crossref]

8. Sápi Z (2014) Pathology of soft tissue sarcomas. Hungarian Oncology 58: 11-23.

9. Renzi S, Anderson ND, Light N, Gupta A (2018) Ewing like sarcoma: An emerging family of round cel sarcomas. J Cell Physiol 234: 7999-8007. [Crossref]

10. Lindberg: Soft tissue tumors (Diagnostic pathology), Elsevier. 2016.

11. Miettinen M, Sobin LH, Sarlomo-Rikala M (2000) Immunhistochemical spectrum of GISTs at different sites and their differential diagnosis with a reference to CD-117 (KIT). Mod pathol 13: 1134-1142. [Crossref]

12. Soslow RA, Bhargava V, Warmke RA (1997) MIC-2, TdT, bcl-2, CD34 expression in paraffin-. embedded high-grade lymphoma/acute lymphoblastic leukemia distinguish between distinct clinicopathologic entities. Hum Pathol 28: 1158-1165.

Copyright: (C2020 Zöllei I. This is an open-access article distributed under the terms of the Creative Commons Attribution License, which permits unrestricted use, distribution, and reproduction in any medium, provided the original author and source are credited. 